\title{
Parallel microgenetic algorithm design for photonic crystal and waveguide structures
}

\section{J. Cai}

L. Li

Gregory P. Nordin

nordin@byu.edu

J. Jiang

Follow this and additional works at: https://scholarsarchive.byu.edu/facpub

Part of the Electrical and Computer Engineering Commons

\section{Original Publication Citation}

J. Jiang, J. Cai, G. P. Nordin, and L. Li, "Parallel microgenetic algorithm design for photonic crystal and waveguide structures," Opt. Lett. 28(23), pp. 2381-2383 (23)

\section{BYU ScholarsArchive Citation}

Cai, J.; Li, L.; Nordin, Gregory P.; and Jiang, J., "Parallel microgenetic algorithm design for photonic crystal and waveguide structures" (2003). Faculty Publications. 1046.

https://scholarsarchive. byu.edu/facpub/1046

This Peer-Reviewed Article is brought to you for free and open access by BYU ScholarsArchive. It has been accepted for inclusion in Faculty Publications by an authorized administrator of BYU ScholarsArchive. For more information, please contact ellen_amatangelo@byu.edu. 


\title{
Parallel microgenetic algorithm design for photonic crystal and waveguide structures
}

\author{
Jianhua Jiang, Jingbo Cai, Gregory P. Nordin, and Lixia Li \\ Laboratory for Integrated Computing and Optoelectronic Systems, University of Alabama in Huntsville, Huntsville, Alabama 35899
}

Received June 20, 2003

\begin{abstract}
We have developed a powerful parallel genetic algorithm design tool for photonic crystal and waveguide structures. The tool employs a small-population-size genetic algorithm (microgenetic algorithm) for global optimization and a two-dimensional finite-difference time-domain method to rigorously design and optimize the performance of photonic devices. We discuss the implementation and performance of this design tool. We demonstrate its application to two photonic devices, a defect taper coupler to connect conventional waveguides and photonic crystal waveguides, and a sharp $90^{\circ}$ waveguide bend for low index contrast waveguides. (C) 2003 Optical Society of America

OCIS codes: $230.3120,230.7370,230.3990,250.5300$.
\end{abstract}

Optimization of photonic components in which the minimum feature size is of the order of a wavelength or smaller poses significant computational challenges because of the need for rigorous solution of Maxwell's equations with computationally intensive numerical tools such as the finite-difference time-domain (FDTD) method $^{1}$ and finite-element methods. ${ }^{2}$ Photonic crys$\mathrm{tal}^{3}$ and compact waveguide structures ${ }^{4}$ are examples of devices that are often designed by manual scanning of desired performance metrics as a function of device parameters. This approach not only can be tedious and time consuming but also can leave the device not fully optimized and overlook novel, unanticipated solutions.

In this Letter we report the development of a powerful parallel genetic algorithm (GA) design tool for photonic crystal and waveguide structures. Our tool employs a small-population-size genetic algorithm [a microgenetic algorithm $\left.{ }^{5}(\mu \mathrm{GA})\right]$ as a global optimization method and a two-dimensional (2D) FDTD method for rigorous evaluation of the performance of photonic devices. $\mathrm{GAs}^{6-8}$ are patterned after natural evolutionary processes, e.g., survival of the fittest. Because of their robustness and efficiency, interest in GAs has grown rapidly in recent years among researchers in many disciplines, including photonics design. However, only a few successful attempts at photonicdevice optimization have been reported, ${ }^{9-11}$ mainly because conventional GAs (CGAs; Ref. 6) use a relatively large population size $(\sim 100)$. This large size results in an unmanageable computational burden when the CGAs are tied to computationally intensive rigorous electromagnetic methods such as those mentioned above, even for today's supercomputers and Linux Beowulf clusters. To overcome this difficulty we recently applied a $\mu \mathrm{GA}$ with a small population size (usually only five individuals) combined with a $2 \mathrm{D}$ FDTD method to successfully optimize finite-aperture diffractive optical elements. ${ }^{12}$ Here we report further development of this $\mu \mathrm{GA}$ optimization tool by parallelizing the $\mu \mathrm{GA}$ code with the MPICH library, a popular Message-Passing Interface (MPI) implementation, for Linux clusters, as discussed below. We also added a new type of variable to the $\mu \mathrm{GA}$, dependent variables that can be varied as a function of one or more independent variables. We found that dependent variables provide much more flexibility for complicated geometry control, which is often required in the design of complex photonic devices.

The key strategy of a $\mu$ GA for achieving small population size is that it consecutively replaces the whole population (except for the best individual) with a new population when the old one converges. This is referred to as elitism ${ }^{6}$ and restart. ${ }^{5}$ From the point of view of optimization, the $\mu \mathrm{GA}$ searches for a global optimum by successively finding local optima through a series of CGA processes executed with small populations. A $\mu \mathrm{GA}$ involves the same genetic operations as a CGA, which include chromosomal coding of the problem parameters, definition and evaluation of the fitness function, selection methods for survival of the fittest individuals, and breeding of a new generation of individuals. Because these genetic operators are well documented in the literature, the reader is referred to Refs. 5-7 for a detailed explanation. Reference 8 gives some information on how to apply a CGA to several classes of electromagnetic optimization problem. Definition of the fitness function is crucial to the success of any GA. In our $\mu \mathrm{GA}$ the fitness function can be flexibly defined by either a weighting function or a target function approach, depending on the nature of the photonic device that is being designed. The design examples include the use of both approaches. Multiple detectors in the 2D FDTD space can also be used in both the spatial and the spectral domains, so multifunctional devices can be designed.

An attractive property of the $\mu \mathrm{GA}$ compared with other global optimization methods, such as simulated annealing, ${ }^{13}$ is that it is computationally parallel and is therefore well suited to be implemented on inexpensive Linux Beowulf clusters. The parallelism can easily be achieved by distribution of the computation of each individual in the population to a slave node such that the computation time for each generation is essentially the same as for each individual, provided that the parallelization overhead is negligible. To this end, the MPICH library is used for code parallelization. For 
hardware, we set up one 32-node and one 48-node cluster, each of which uses an inexpensive off-the-shelf PC with a $2.0-\mathrm{GHz}$ CPU and 1.0 GB of RAM.

To demonstrate the design capability of our photonic design tool, we first consider the important problem of high-efficiency coupling between photonic crystal waveguides (PhCWGs) and conventional waveguides (CWGs). In particular, we consider the pair of defect taper couplers (DTCs) shown in Fig. 1 to connect a PhCWG to input and output CWGs. This geometry is similar to that of Sanchis et al. ${ }^{14}$ except that both the CWG and the PhCWG are single mode and our design goal is to maximize the optical transmission of the system into the single mode supported by the output waveguide. The design problem is therefore more difficult ${ }^{15}$ than for the multimode CWG described in Ref. 14. We consider silica CWGs with a $2-\mu \mathrm{m}$-wide core of refractive index 1.474 embedded in cladding with index 1.445 (2\% index difference) and light with a free-space wavelength of $1.55 \mu \mathrm{m}$. The photonic crystal structure is a $2 \mathrm{D}$ triangular array of silicon posts embedded in the CWG core material with a lattice constant, $a$, of $0.465 \mu \mathrm{m}$. The $\mathrm{Si}$ posts have radius $0.2 a$ and refractive index 3.45. This photonic crystal has a bandgap at a normalized frequency range $a / \lambda_{0}=0.2647-0.3565$ for TM polarization (electric field pointed out of the plane) as calculated with MIT Photonic-Bands software. ${ }^{16}$ We form the single-mode PhCWG by removing a row of $\mathrm{Si}$ posts in the $\Gamma K$ direction.

First we consider the case in which no defect posts are present. The transmission efficiency for TM polarization is only $13.5 \%$ (i.e., a fraction of optical power launched into the input waveguide that is transmitted into the optical mode supported by the output waveguide). Introduction of Si defect posts into the taper region can help to improve the coupling between the CWGs and the PhCWG, but the difficulty lies in determining what defects to introduce and where to place them. In Ref. 14 the authors presented a simple and intuitive optimization process to achieve a design when there are two on-axis defects. However, the resultant design is not necessarily optimal. Moreover, it is almost impossible to apply their process to more complicated defect taper coupler (DTC) situations with multiple off-axis defects.

To illustrate the utility of the $\mu \mathrm{GA}$ as a design tool, we consider six off-axis elliptical Si defect posts in each DTC, of which three are independently optimized and the others are symmetrically arranged about the waveguide's symmetry axis. Using dependent variables, we make the defects in the output coupler identical to those in the input coupler. The center coordinates and the lengths of the major and minor axes of the independent defect posts are encoded as variables such that there are a total of 12 independent variables in the $\mu \mathrm{GA}$ optimization. The lengths of the major and minor axes can be independently varied from $0.1 a$ to $0.5 a$, and the $x$ and $y$ center coordinates can be varied from 1.0 to $3.5 \mu \mathrm{m}$. The fitness function is defined by a target function in the form

$$
F=\sum_{i} \operatorname{abs}\left(P_{i}-P_{i}{ }^{M}\right)
$$

where $P_{i}$ is the magnitude of the actual Poynting vector on the detector plane and $P_{i}{ }^{M}$ is the magnitude of the Poynting vector of the mode supported by the CWG. Symmetry about the $x$ axis is exploited in the FDTD calculation to reduce the computation time. Each $\mu$ GA generation takes $\sim 15$ min to run, with a square Yee cell resolution of $\lambda_{0} / 120$. Figure 2 is a typical $\mu \mathrm{GA}$ convergence curve, which clearly shows the rapid convergence of our $\mu \mathrm{GA}$. Figure 3 shows a plot of the geometry of the best design obtained by $\mu \mathrm{GA}$, along with the distribution of the magnitude squared of the time-averaged electric field amplitude calculated by the FDTD method. The transmission efficiency of this DTC is $93.2 \%$, with a backreflection of $2.49 \%$. The optimized parameters for the three

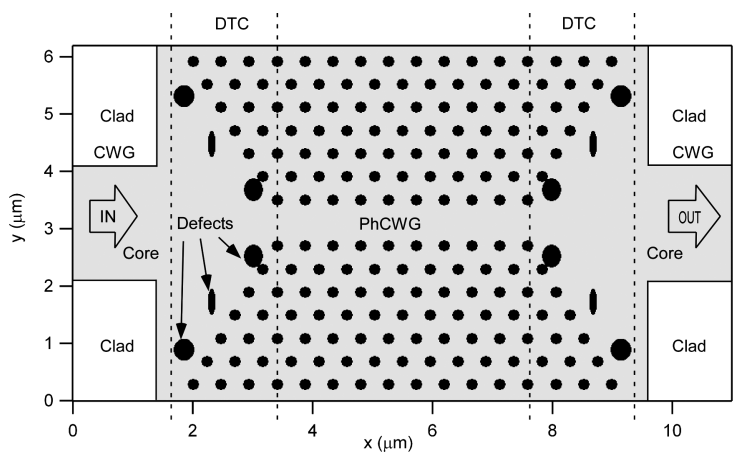

Fig. 1. Schematic view of a DTC for input and output coupling between a single-mode CWG and a single-mode PhCWG.

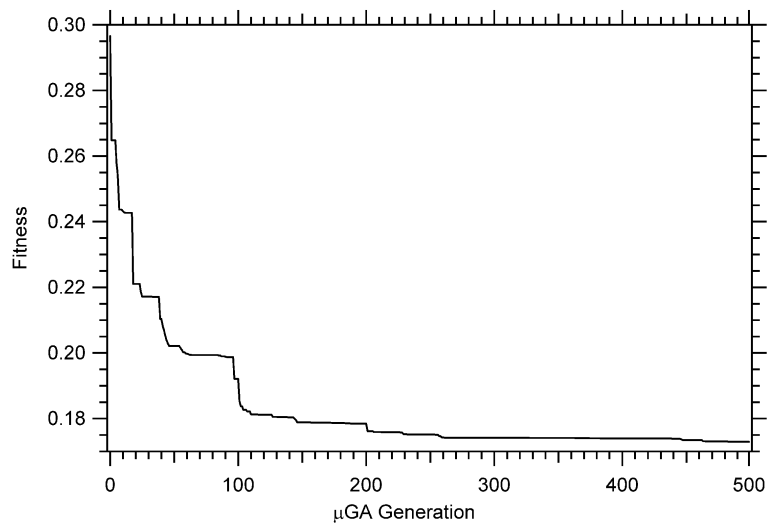

Fig. 2. Typical convergence curve of a $\mu \mathrm{GA}$.

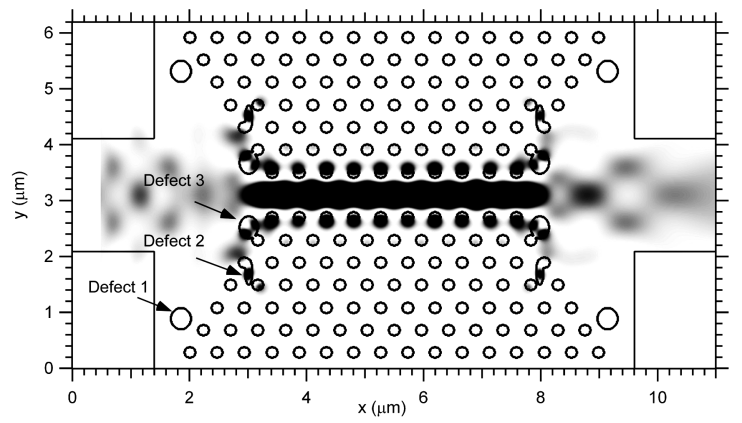

Fig. 3. DTC with three $\mu$ GA-optimized off-axis Si defect posts: geometry and magnitude squared of the electric field amplitude. 
Table 1. Parameters of Three Optimized Si Defect Posts

\begin{tabular}{ccc}
\hline $\begin{array}{c}\text { Defect } \\
\text { Number }\end{array}$ & $\begin{array}{c}\text { Center Coordinates } \\
(x, y)(\mu \mathrm{m})\end{array}$ & $\begin{array}{c}\text { Half-Major Axis } \\
(x, y)(\mu \mathrm{m})\end{array}$ \\
\hline I & $(3.0116,2.5217)$ & $(0.1572,0.1986)$ \\
II & $(3.0204,1.7194)$ & $(0.0641,0.2253)$ \\
III & $(1.8603,0.8916)$ & $(0.1721,0.1896)$ \\
\hline
\end{tabular}

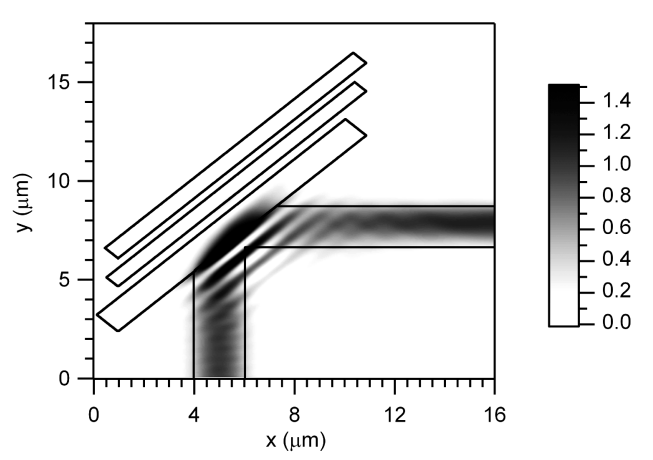

Fig. 4. Geometry and magnitude squared of the electric field amplitude of an optimized three-layer air-trench bend with $97.6 \%$ bend efficiency for TM polarization.

independent defect posts are listed in Table 1. The minimum feature size is approximately $130 \mathrm{~nm}$.

Our second photonic device design example is a multiple-layer air-trench $90^{\circ}$ bend for a low-index contrast waveguide. The objective is to design a set of air trenches that efficiently redirect light from the input waveguide to the output waveguide (see Fig. 4). As discussed in Ref. 17, one can understand the operation of air interface structures by referring to the angular spectrum of the waveguide mode in relation to the critical angle for total internal reflection. For a $90^{\circ}$ bend, the set of air trenches must carefully balance the trade-off between frustrated total internal reflection of angular spectrum components that would normally undergo total internal reflection at a single air interface and increase the reflection of the rest of the angular spectrum components.

We consider a single-mode CWG with the same parameters as our first example and a set of three $14-\mu \mathrm{m}$-long air trenches. The $y$ coordinate and the thickness of the trenches are set to be variables, so there are six variables in this optimization. A rectangular weighting function is used to maximize the power directed into the output waveguide:

$$
F=\sum_{i} C \operatorname{rect}\left(x_{i} / W\right) P\left(x_{i}\right)
$$

where $W$ is the width of the waveguide and $C$ is an arbitrary weighting factor. A much coarser Yee cell size $\left(\lambda_{0} / 40\right)$ can be used in the FDTD simulation because the largest refractive index is much smaller than in the first example, and, because this dramatically reduces the computational time, a sequential version of our design tool is used here. Each $\mu \mathrm{GA}$ generation takes $\sim 14.5$ min on a Dell Dimension 8100 computer with a 1.3-GHz CPU and 384-MB RAM. Figure 4 shows an optimized device along with the magnitude squared of the electric field amplitude. The fraction of the incident light (1.55- $\mu \mathrm{m}$ wavelength and TM polarization) that is redirected into the output waveguide is $97.6 \%$. The optimized thicknesses of the three air trenches are $1.19,0.64$, and $0.74 \mu \mathrm{m}$, and the two spacings are 0.42 and $0.36 \mu \mathrm{m}$.

In summary, we have developed a powerful parallel design tool for photonic crystal and waveguide structures that employs a small-population-size genetic algorithm as a global optimization method and a two-dimensional finite-difference time domain as the rigorous electromagnetic computational engine. By adopting the $\mu \mathrm{GA}$ we shrink the population size of a conventional genetic algorithm from $\sim 100$ to merely 5 , which in turn reduces the number of required FDTD computations by more than an order of magnitude. We recently finished parallelizing the 2D FDTD code with the MPI interface and are in the process of linking the code with the parallel $\mu \mathrm{GA}$ computational kernel. We expect this fully parallelized design tool to successfully address much larger photonic-device design problems in a reasonable amount of time.

This work was supported by Defense Advanced Research Projects Agency grant N66001-01-1-8938 and National Science Foundation grant EPS-0091853. J. Jiang's e-mail address is jiangj@email.uah.edu.

\section{References}

1. A. Taflove, Computational Electrodynamics: The Finite-Difference Time-Domain Method (Artech House, Norwood, Mass., 1995).

2. K. Okamoto, Fundamentals of Optical Waveguides (Academic, San Diego, Calif., 2000).

3. E. Yablonovitch, Phys. Rev. Lett. 58, 2058 (1987).

4. R. L. Espinola, R. U. Ahmad, F. Pizzuto, M. J. Steel, and R. M. Osgood, Jr., Opt. Express 8, 517 (2001), http://www.opticsexpress.org.

5. K. Krishnakumar, Proc. SPIE 1196, 289 (1989).

6. D. E. Goldberg, Genetic Algorithm in Search, Optimization, and Machine Learning (Addison-Wesley, Reading, Mass., 1989).

7. Z. Michalewicz, Genetic Algorithms + Data Structures = Evolution Programs (Springer-Verlag, Berlin, 1996).

8. Y. Rahmat-Samii and E. Michielssen, Electromagnetic Optimization by Genetic Algorithms (Wiley, New York, 1999).

9. E. G. Johnson and M. A. G. Abushagur, J. Opt. Soc. Am. A 12, 1152 (1995).

10. G. Zhou, X. Yuan, P. Dowd, T. Lam, and Y. Chan, J. Opt. Soc. Am. A 18, 791 (2001).

11. Q. Wang, J. Lu, and S. He, Appl. Opt. 41, 7644 (2002).

12. J. Jiang and G. Nordin, Opt. Express 7, 237 (2000), http://www.opticsexpress.org.

13. S. Kirkpatrick, C. D. Gellatt, Jr., and M. P. Vecchi, Science 220, 671 (1983).

14. P. Sanchis, J. Martí, J. Blasco, A. Martínez, and A. García, Opt. Express 10, 1391 (2002), http:// www.opticsexpress.org.

15. A. Mekis and J. D. Joannopoulos, IEEE J. Lightwave Technol. 19, 861 (2001).

16. See http://ab-initio.mit.ed/mpb.

17. L. Li, G. P. Nordin, J. M. English, and J. Jiang, Opt. Express 11, 282 (2003), http://www.opticsexpress.org. 